\title{
Advanced or Metastatic Gastrointestinal Stromal Tumors: Systemic Treatment Options
}

\author{
MEGAN V. CARAM, MD AND SCOTT M. SCHUETZE, MD, PhD* \\ Division of Hematology/Oncology, Department of Internal Medicine University of Michigan, Michigan
}

\begin{abstract}
Gastrointestinal stromal tumor (GIST), the most common sarcoma arising in the gastrointestinal tract, typically expresses the tyrosine-kinase receptor, C-KIT, and contains activating mutation in the $c$-kit or platelet-derived growth factor receptor ( $p d g f r)$ gene. Recently, development of small molecules that inhibit the kinase activity of mutant C-KIT and PDGFR proteins has radically changed treatment and prognosis of patients diagnosed with advanced GIST as this molecularly "targeted" therapy has demonstrated remarkable high-level of activity in this disease. J. Surg. Oncol. 2011;104:888-895. () 2011 Wiley Periodicals, Inc.
\end{abstract}

\section{KeY WoRDs: GIST; imatinib; sunitinib}

\section{INTRODUCTION}

Gastrointestinal stromal tumors (GISTs) have emerged as a distinct entity over the past few decades as tumor identification has improved and GISTs are now being readily identified by specific immunohistochemistry. As noted in the National Comprehensive Cancer Network (NCCN) Task Force Report, the world-wide incidence of GIST is thought to be approximately 10 cases per million persons each year; an incidence estimated based on reported annual incidence ranging between 6.5 and 14.5 cases per million [1]. Many advances in the treatment of GISTs have emerged over the past decade following the recognition of this distinct entity with the greatest impact on management of advanced or metastatic disease achieved by the introduction of imatinib and other tyrosine kinase inhibitors. Before imatinib, the median survival of patients diagnosed with advanced GIST was 10-17 months [2]. In this day and age, patients with advanced disease treated with tyrosine kinase inhibitors are now living $>50$ months [3]. This review will help to clarify some of the latest advances and the current standard of care for treating advanced GIST in this era.

GISTs are malignant tumors that have been found to arise in all areas of the gastrointestinal tract from the esophagus to the rectum and may originate from the pacemaker cells of the GI tract, the interstitial cells of Cajal. The most common site of origin is the stomach $(60 \%)$, followed by small intestine (30\%), duodenum (5\%), colon/ rectum $(<5 \%)$, and esophagus $(<1 \%)$ [1]. GISTs can rarely originate in the omentum, mesentery, or retroperitoneum; extra-gastrointestinal GISTs seem to behave in an aggressive way, similar to small bowel GISTs [4]. GISTs have the potential to metastasize to distant sites, but usually spread locally to peritoneal surfaces, the omentum and to liver. GISTs rarely metastasize to the lung unlike other sarcomas [5]. Lymph node and bone involvement, and spread outside of the abdominal cavity are exceedingly uncommon compared to GIST propensity for local spread [6]. Great care must be undertaken when excising GISTs as these tumors also have the potential to seed areas in the abdomen following peri-operative tumor rupture. Tumor seeding during needle biopsy to establish the diagnosis may also occur [1].

Prior to describing the treatment of advanced GIST, it is prudent to first review some of the pathophysiology of the disease since many of the drug treatments interact with proteins important in the genesis of GIST. Certain mutations that activate specific proteins have been found to be involved in GIST pathogenesis. These activated proteins are now targets for effective therapies in GIST treatment. Approximately $90 \%$ of GISTs have activating mutations in genes for the transmembrane receptors C-KIT or platelet derived growth factor receptor-alpha (PDGFR-A) [7]. The majority of GISTs $(85 \%)$ contain activating mutations in $c$-kit, $5 \%$ contain mutations in $p d g f r-a$, and the remainder of GISTs do not contain identifiable mutations in either of these two receptor kinases, otherwise known as "wild-type" GIST. Mutations in exon 11 of $c$-kit are the most common and convey a high degree of sensitivity to first-line therapy with imatinib, which will be discussed in greater detail later in this review [1]. Exon $9 c$-kit mutations are the second most common mutations in this transmembrane receptor and are associated with a different sensitivitiy to imatinib and to other tyrosine kinase inhibitors. Mutations in exons 13, 14, 17, and 18 of $c$-kit have also been described, though less commonly, and in some cases have been associated with primary or secondary resistance to imatinib. Mutations in exons 12, 14, and 18 in $p d g f r-a$ have been found to be the etiology for many GISTs that do not have activating mutations in $c$-kit and carry with them their own prognosis based on responsiveness to drugs currently available [8]. Some wild-type GISTs without mutations in $c$-kit or pdgfr- $a$ have increased expression of insulin-like growth factor-1 receptor (IGF1-R) [9] and a potentially worse prognosis as this phenotype is associated with a higher mitotic index, larger size, and higher rate of recurrence and metastasis [10].

Patients with certain genetic disorders such as von Recklinghausen's disease, familial GIST, and Carney-Stratakis syndrome make up a small percentage of the GIST population. Another disorder, Carney's triad, is a non-inherited condition that has been described in less than 100 GIST patients world-wide, and is associated with

*Correspondence to: Scott M. Schuetze, MD, PhD, C342 Med Inn, SPC 58481500 E Medical Center Dr. Ann Arbor, MI 48109-5848. Fax: 734647-7279. E-mail: scotschu@med.umich.edu

Received 22 November 2010; Accepted 14 March 2011

DOI 10.1002/jso.21930

Published online in Wiley Online Library (wileyonlinelibrary.com). 
extra-adrenal paragangliomas and pulmonary chondromas [1]. GISTs typically arise in the stomach and follow a prolonged and indolent course in patients with Carney's triad. Patients with von Recklinghausen's disease, or neurofibromatosis type-1 (NF-1), have a predisposition for GIST, but more commonly develop gliomas and neurofibromas as part of the NF-1 syndrome. The development of GIST in NF-1 is thought to be due to a loss of the gene encoding neurofibromin that predisposes the interstitial cells of Cajal to hyperplasia [11]. GISTs associated with NF-1 may also be more indolent and less aggressive than sporadic GISTs [12] and are typically found in the small bowel. Familial GISTs are part of a syndrome with germline mutations in $c$-kit or $p d g f r-a$, and are found to be associated with cutaneous hyperpigmentation, primarily on the hands and perineum, and other gastrointestinal problems such as dysphagia, irritable bowel syndrome, diverticular disease, and others [13]. These patients usually present with multiple primaries, and again the disease is often more indolent than sporadic GISTs [1]. Carney-Stratakis syndrome is another inherited germline disorder that involves a dyad of the tumors seen in Carney's triad. Patients with GIST in the setting of this syndrome typically are diagnosed with disease in the stomach. Even though GIST arising in a non-familial GIST syndrome usually expresses the C-KIT protein, $c$-kit and pdgfr-a genes are unmutated, and patients who develop GIST in this setting have a lower likelihood of tumor responding to kinase inhibitors targeting activated C-KIT and PDGFR proteins.

\section{RISK STRATIFICATION}

For patients initially diagnosed with primary GIST without evidence of metastases, tumor location, size, and mitotic rate have been found to be associated with a higher risk for recurrence and the development of metastases after surgical resection [14]. These three predominant factors were used to develop risk stratification by a National Cancer Institute consensus conference and subsequently a prognostic nomogram by Gold, et al., with the purpose of providing guidance for selecting patients who may potentially benefit from adjuvant treatment after primary resection. Tumor size $>10 \mathrm{~cm}$, mitotic index $>5$ per 50 high-power fields (HPF) of view and small intestine as the site of origin were associated with the highest probability for recurrence, while tumor size $<5 \mathrm{~cm}$, mitotic index $<5$ per $50 \mathrm{HPF}$ and stomach as the site of origin were associated with the lowest probability for recurrence using a point system that predicted 2 and 5 year progression-free survival [14]. The use of adjuvant therapy for high-risk GIST following resection will be discussed in a different section, though understanding which aspects of this disease portend a poorer prognosis can be helpful in appreciating the development of advanced disease.

\section{THERAPY FOR ADVANCED GIST}

Molecular targeted therapies have become important and exciting in the treatment of cancers for which specific mutations thought to be crucial for pathogenesis have been discovered. However, prior to the discovery of these pathologically important mutations and the development of targeted therapy directed at these mutated proteins, drug therapy for advanced GIST was non-specific and disappointingly ineffective. Here, we review a few of the chemotherapies studied in advanced GIST for historical perspective.

\section{Historical Perspective}

Systemic chemotherapy given as either single agent or as a combination of agents that are known to be of benefit in other sarcomas such as leiomyosarcoma have been mostly inactive in patients with GIST. One study from Mayo Clinic published in 1999 evaluated the response to dacarbazine, mitomycin $\mathrm{C}$, doxorubicin, cisplatin, and growth factor support in patients with GIST or leiomyosarcoma and reported that one out of 21 patients with GIST had a partial response (4.8\%) compared to $67 \%$ of patients with leiomyosarcomas [15]. DeMatteo, et al. [16] reviewed a series of trials evaluating different combinations of chemotherapeutic drugs such as doxorubicin, dacarbazine, ifosfamide, cisplatin, and others to treat patients with advanced GISTs and reported partial responses of between 0 and $15 \%$ among a total of 266 patients, with a mean partial response rate of $8.3 \%$. Therefore, traditional cytotoxic chemotherapy has poor activity against GIST providing increased motivation for the development of novel agents.

Intraperitoneal (IP) chemotherapy for the treatment of GIST was proposed initially by Berthet, et al. [17] in order to reduce peritoneal recurrence after surgical resection of GIST and other intra-abdominal sarcomas. Because GISTs tend to spread to serosal surfaces by regional metastasis, this strategy was thought to be promising. Investigators have used IP instillation of cisplatin with doxorubicin or single-agent mitoxantrone in an effort to limit systemic toxicity and control peritoneal disease. Eilber et al. [18] looked specifically at IP mitoxantrone in 33 patients with recurrent GIST with either limited peritoneal involvement, or peritoneal and liver involvement. All patients underwent surgical resection of all gross disease in the abdomen and those with liver involvement underwent treatment (resection, chemoembolization, or cryotherapy) of the liver lesions. All 33 patients received 4-6 cycles of IP mitoxantrone. The 33 patients were compared to 13 patients who only had surgery without IP chemotherapy. Among the patients treated with surgery alone, disease recurred in all 13 and only $23 \%$ were alive at a mean follow-up time of 19 months. Among the patients treated by surgical resection followed by IP therapy, disease recurred in $85 \%$ of patients at a median of 11 months, however the other $15 \%$ were disease free at a mean follow-up of 39 months, and $27 \%$ were alive at a mean followup of 43 months. This small, non-randomized study suggests that a small proportion of carefully selected patients may have long-term benefit from IP chemotherapy following surgical resection. However, in the era of available tyrosine kinase inhibitor therapy, treatment of GIST with chemotherapy has been supplanted.

Radiation therapy has been found to have limited capacity to affect GISTs and isn't routinely used for advanced disease because metastases usually affect more than one area. Sometimes radiation can be used for palliation of a bulky tumor if it causes pain or to control bleeding. However, literature using this method of control is limited; radiation has an unknown benefit in advanced GIST outside of palliating symptoms [16].

\section{Imatinib Use in GIST}

Imatinib is a small molecule tyrosine kinase inhibitor that was developed in the mid-1990s based on its ability to inhibit the ABL and $\mathrm{BCR} / \mathrm{ABL}$ tyrosine kinases. It was first approved for treatment of the hematologic disorder chronic myelogenous leukemia because of the drugs remarkable efficacy. Imatinib was also found to inhibit the transmembrane tyrosine kinase receptors C-KIT and PDGFR alpha and beta. In 1998, Hirota, et al. [19] discovered that some patients with GISTs had mutations in the C-KIT tyrosine kinase that aberrantly activated the kinase in tumor tissue. Since the discovery of these activating mutations in GISTs, it was hypothesized that aberrant tyrosine kinase activity may be an important etiologic factor in the development of GIST. Investigation of the impact of therapy specifically inhibiting the tyrosine kinase activity in GIST has been rapid in development considering the limited efficacy of other systemic therapy. In 2000, Joensuu, et al. [20] used imatinib to treat 
advanced GIST in a single patient with widely metastatic disease. This single patient achieved a major objective disease response detected by magnetic resonance imaging and positron emission tomography that persisted for more than 18 months. After 4 weeks, biopsies of GIST metastasis in this patient revealed a histology of mostly myxoid degeneration and fibrosis, suggesting induced apoptosis caused by imatinib. Because of the success seen in this patient, and the desperation in the medical community to discover an active treatment for metastatic GIST, subsequent large-scale studies using imatinib were developed and completed shortly thereafter.

\section{Treatment of Advanced/Metastatic Disease}

Blanke, et al. [3] conducted a prospective, randomized, phase II trial (B2222) of patients with advanced GIST assigned to receive either $400 \mathrm{mg}$ or $600 \mathrm{mg}$ daily of imatinib. Patients who showed tumor response to imatinib were offered participation in an extension trial that followed them for four more years on daily imatinib. Patients (147) were initially enrolled in the core study with 67 patients completing the study. Fifty-six of the 67 patients completing the core study were entered into the extension study. Out of the initial 147 patients enrolled, $68.1 \%$ of patients achieved a partial or complete response, with an additional $15.6 \%$ of patients maintaining stable disease without progression over a follow-up of up to 71 months.

After impressive results were seen with imatinib in the randomized phase II study, subsequent larger scale confirmatory trials were done to evaluate response and toxicity rates. In a trial by Verweij et al. [21] comparing $400 \mathrm{mg}$ versus $800 \mathrm{mg}$ of imatinib daily in patients with metastatic GIST, 946 patients were enrolled, randomized and followed on an intent-to-treat basis with cross-over allowed from the lower to higher dose treatment following progression of disease. Over a median follow-up of 760 days, there were no significant differences between the groups in rates of objective response, stable disease, or tumor progression. Fifty percent of the patients in the $400 \mathrm{mg}$ group had a partial or complete response, compared with $54 \%$ of patients in the $800 \mathrm{mg}$ group. Thirty-two percent of patients in the $400 \mathrm{mg}$ and $800 \mathrm{mg}$ group had stable disease, and $13 \%$ of patients in the $400 \mathrm{mg}$ group compared with $9 \%$ of patients in the $800 \mathrm{mg}$ group had tumor progression. A phase III study performed in North America (S0033) that was published in 2008 also compared $400 \mathrm{mg}$ versus $800 \mathrm{mg}$ daily dosing of imatinib in 746 patients and found similar results to the contemporaneous European Organization for Research and Treatment of Cancer (EORTC) trial for objective response, stable disease, and progression [22]. This phase III trial also looked at progression-free survival and overall survival between the two groups and found no statistically significant difference. However, there was a difference in toxicity, with reported higher rates of grade 3,4 , and 5 toxicities in the $800 \mathrm{mg}$ group. Therefore, the conclusion of the study was that $400 \mathrm{mg}$ daily dosing is an appropriate starting dose for patients with metastatic GIST, with less risk for toxicity than the higher starting dose [22]. The Food and Drug Administration approved the marketing of imatinib at a starting dose of $400 \mathrm{mg}$ daily for treatment of patients with advanced or relapsed GIST.

In patients whose disease progressed on imatinib $400 \mathrm{mg}$ daily, cross-over was allowed to the $800 \mathrm{mg}$ dose in S0033 and $31 \%$ of the patients who crossed over had stabilization of disease growth with a median gain in progression free survival of 5 months following cross-over [22]. Because of a reasonable chance for tumor control following increase in imatinib dose, the Soft Tissue Sarcoma Guideline Panel from the NCCN recommended dose escalation to $800 \mathrm{mg}$ a day in patients whose disease has progressed on the $400 \mathrm{mg}$ daily dose as long as side effects from imatinib are easily manageable [23].

\section{Neoadjuvant Imatinib Therapy for Locally Advanced Disease}

Because imatinib has been shown to be effective against metastatic GIST, naturally investigators evaluated its use in the adjuvant and neoadjuvant setting in an attempt to increase cure rates. A few trials have investigated the perioperative use of imatinib in potentially resectable localized or metastatic disease to demonstrate its safety. However, these trials did not specifically evaluate the effect of pre-operative imatinib on surgical margin outcomes nor demonstrate a significant survival benefit from pre-operative imatinib because all patients received post-operative imatinib for 2 years $[24,25]$. Another hypothesis that was evaluated is that patients with marginally resectable disease, or tumors in locations that pose a high surgical morbidity, would benefit from neoadjuvant (pre-operative) use of imatinib. A retrospective study of 46 patients with locally advanced, recurrent, or metastatic disease demonstrated that neoadjuvant imatinib improved some of the patients' chances for complete resection [26]. The duration of pre-operative imatinib varied by patient, depending on disease response on serial imaging tests. Patients were taken to surgery when their every 2-3 month computed tomography scan ceased to show further reduction in tumor size or showed progression of disease. Eleven of the 46 patients were treated with imatinib for a median of 11.9 months for locally advanced GIST prior to resection of disease. At a follow-up of 19.5 months, all 11 were living and 10 were free of disease. Of the 46 patients, those who experienced a partial tumor response to imatinib had a greater chance for complete resection $(91 \%$ vs. $4 \%, P<0.001)$. A prospective study performed by Hohenberger, et al. [27] in 36 patients with locally advanced or recurrent GISTs evaluated preoperative GIST response to imatinib, the extent of surgery required for tumor excision, morbidity of surgery and local disease recurrence rates. Median tumor shrinkage of about $5 \mathrm{~cm}$ was seen (from a median of $10.5 \mathrm{~cm}$ pre-imatinib to $5.5 \mathrm{~cm}$ immediately pre-op) in 33 patients who underwent a median of 11 months of pre-operative imatinib therapy. Twenty-eight of the 33 patients had complete resection of tumor, and in $83 \%$ the previously "inoperable" disease became operable after neoadjuvant therapy. In regards to morbidity, 21 of 25 patients underwent less morbid surgeries due to tumor response from the neoadjuvant therapy.

Imatinib has not been approved for pre-operative treatment in patients with resectable, localized GIST. However, as imatinib is now occasionally used neoadjuvantly, there have been reports of potentially deleterious effects in some patients treated pre-operatively. The dramatic tumor shrinkage that can occur with imatinib may put some patients at increased risk of severe gastrointestinal or IP bleeding, such as in patients with rare duodenal GISTs [28]. However, patients with large tumors in the stomach may have a decreased risk of intra-operative tumor rupture and easier resection following neoadjuvant imatinib [29]. Therefore, the decision for or against neoadjuvant use of imatinib should be made in a multi-disciplinary setting in order to individualize therapy for each patient's unique tumor anatomy. With good pre-operative response to imatinib, tumors in areas such as the rectum or gastroesophageal junction, that would otherwise require a highly morbid surgery, may be amendable to sphincter- and esophagus-sparing surgeries, respectively.

\section{Dosing of Imatinib}

Whether measurement of imatinib serum levels will be helpful in assessing dose adequacy in patients treated for GIST is controversial. A sub-group of 73 patients from the B2222 study had imatinib levels drawn on days 1 and 29 after beginning either 400 or $800 \mathrm{mg}$ of imatinib daily. In a retrospective analysis, in the group of patients who had a level $<1,100 \mathrm{ng} / \mathrm{ml}$, the time to progression was 
11.3 months as compared to a time to progression of greater than 30 months in the group of patients with a level $>1,100 \mathrm{ng} / \mathrm{ml}$ [30]. There is an ongoing, randomized, prospective trial examining whether an increase in dose for patients found to have imatinib levels $<1,100 \mathrm{ng} / \mathrm{ml}$ will affect outcomes. However, due to the lack of evidence that increasing imatinib dose in patients based on drug levels in serum will alter risk of disease progression, measuring imatinib levels is not recommended.

\section{Duration of Imatinib Therapy}

The duration of imatinib therapy in metastatic GIST has been evaluated in a French Sarcoma Group study randomizing patients with responding or stable disease to interruption of treatment after 1 year and after 3 years of imatinib [31,32]. Out of 182 patients initially enrolled in the trial, 58 of the patients who experienced tumor control for at least 1 year were randomized to treatment interruption or continuation of imatininb. Ninety-one percent of the patients in the treatment interruption arm experienced tumor progression with a mean progression-free survival of 6 months from stopping imatinib compared to a 29 -month progression-free survival in the arm that continued imatinib. However, there was no difference in overall survival between the two groups since the majority $(86 \%)$ of patients who progressed after drug interruption achieved disease control after re-starting imatinib. The same end-points were then evaluated with interruption of imatinib after 3 years. Out of 378 patients with metastatic GIST enrolled in the study, 50 were eligible for randomization after 3 years. The 1-year progression-free survival rate was $92 \%$ in the arm that continued imatinib versus $29.7 \%$ in the arm that had interruption of their treatment. Again, there was no difference in overall survival since all patients in the interruption arm were able to re-gain control of their disease after restarting imatinib. Based on the results of this study, continuation of imatinib for treatment of metastatic GIST is recommended even following complete tumor response to therapy. A brief period without imatinib therapy, for example to allow for elective surgery, does not seem to increase risk of tumor resistance to imatinib.

\section{Metabolism of Imatinib}

Imatinib is metabolized through the hepatic p450 enzyme system by CYP3A4, which is an enzyme central in the metabolism of other medications such as the azoles, rifampin, phenytoin, and warfarin. Inhibitors of CYP3A4 enyzyme activity, such as ketaconazole and grapefruit juice can increase imatinib levels in the serum and possibly cause increased toxicity of imatinib at normal treatment doses. Rifampin and phenytoin are two medications that are known to induce activity of the CYP3A4 enyzme and therefore may lead to rapid metabolism of imatinib reducing efficacy at normal treatment doses [1]. Therefore, care should be taken when prescribing imatinib along with other medications known to affect CYP3A4 activity.

\section{Adverse Effects of Imatinib}

Adverse effects of imatinib that can ensue in most patients are typically tolerable at $400 \mathrm{mg}$ daily dose. Diarrhea, fluid retention, nausea, fatigue, muscle cramps, abdominal pain, and rash are the most common associated side effects of imatinib [33]. Gastrointestinal upset caused by imatinib can sometimes be prevented by taking the drug with food, though supportive medications such as protonpump inhibitors and loperamide for dyspepsia and diarrhea, respectively, can be helpful as well. Fluid retention is a bothersome symptom for many patients, commonly involving the lower extremities and periorbital area, though it can rarely be severe enough to cause ascites and pleural and pericardial effusions. Significant edema can be managed with sodium restriction and furosemide. Muscle cramping is also a particularly annoying adverse effect that is treated by increasing fluid intake, electrolyte replacement beverages, tonic water, and muscle relaxants [1]. Some patients with large bulky tumors, especially duodenal GISTs may have an increased risk for hemorrhage and therefore hemoglobin in these patients should be closely monitored. Myelosuppression and elevated transaminases are less common, and usually resolve with temporary discontinuation of therapy [33]. If leukopenia or severe neutropenia occurs, the current guidelines recommend holding imatinib until blood counts recover the then re-initiating therapy at the former dose [1]. Anemia that develops in patients on long-term imatinib therapy can be caused by iron deficiency, B12 deficiency, folate deficiency, and suppression of hematopoiesis by imatinib [1]. Rarely, severe adverse effects such as acute myelogenous leukemia (AML) and congestive heart failure (CHF) have been reported [34,35]. Discontinuation of therapy in the setting of severe adverse events such as AML and CHF is warranted along with initiation of treatment for these respective conditions.

\section{Resistance to Imatinib}

Imatinib mesylate is a selective inhibitor of a number of tyrosine kinsases, including ABL, BCR/ABL, KIT, and PDGFR. C-KIT is the most commonly mutated tyrosine kinase seen in GIST, and therefore inhibiting C-KIT activity to control the growth of GIST is an important goal of treatment. A subset of GISTs that do not harbor a mutation in $c$-kit typically have either $p d g f r-a$ activating mutations or are considered "wild-type" GISTs with no identifiable mutations in these genes. Mutations in $c$-kit and pdgfr-a appear to be mutually exclusive in that the presence of mutant $c$-kit excludes mutation in $p d g f r-a$ in the same tumor and vice versa. There are different types (point mutations, deletions, insertions) and locations of mutations within the $c$-kit and pdgfr- $a$ genes; mutations in exons 11 and 9 of c-kit comprise approximately $70 \%$ and $15 \%$, respectively, of primary mutations in GISTs [7,36]. Mutations in $c$-kit exons 13 or 17 are much less common. A small proportion of activating mutations seen in GISTs are in exon 12 or 18 of the $p d g f r-a$ gene (between 2 and 5\%) [37,38]. About $10 \%$ of GISTs are typically denoted as "wild-type" since no readily identifiable activating mutation in the transmembrane receptor kinase $c$-kit or pdgfr-a genes are found.

Among the activating mutations in exon 11 of $c$-kit, the most common mutation observed was an in-frame deletion of a portion of the juxtamembrane domain sometimes accompanied by an insertion or a point mutation [7]. Most of the exon 11 mutations in $c$-kit occur between codons 550 and 561 [39]. In exon 9 of $c$-kit, the activating mutations seen have been in-frame duplications resulting in an insertion, usually at codon 502 and infrequently at codon 506 [7]. The most frequently observed activating mutations in $p d g f r-a$ is a point mutation in the kinase activation loop coded in exon 18 resulting in an amino acid change at position 842 (D842V), and a point mutation in the juxtamembrane domain coded in exon 12 (V561D) [7].

The responsiveness of GISTs to imatinib has been observed to differ depending on which mutation is present. Thirty-seven tumor specimens taken from patients being treated with imatinib in an EORTC phase I or II trial were evaluated for mutations by extracting DNA and using PCR to amplify the different areas of interest from the $c$-kit and pdgfr-a genes. This study found that $50 \%$ of patients with mutations in exon 11 of $c$-kit were free from disease progression at 104 weeks of therapy, compared to $25 \%$ of the patients with $c$-kit exon 9 mutations, $0 \%$ of the patients with pdgfr-a mutations and $33 \%$ of patients with "wild-type" GIST. The number of samples in this study were small, though statistical significance for improved progression-free survival with a $P$-value of 0.03 was observed for patients carrying $c$-kit mutations compared to all other patients. Another study by Heinrich, et al. [37] evaluated the GIST 
genotypes from patients treated in the phase III SWOG S0033 trial They observed the median time to tumor progression for patients with mutations in c-kit exon 11, c-kit exon 9, and "wild-type" GISTs to be 24.7, 16.7, and 12.8 months, respectively. Median overall survival was $60.0,38.4$, and 49.0 months for patients with $c$-kit exon 11, exon 9, and "wild-type" GISTs, respectively. This study did not detect a significant difference in time to tumor progression or overall survival for patients with $c$-kit exon 9 mutations treated with $400 \mathrm{mg}$ versus $800 \mathrm{mg}$ daily dose of imatinib, but a different study by Debiec-Rychter, et al. [36] detected a statistically significant $(P=0.0013)$ difference in progression-free survival in patients with exon 9 mutations treated with $400 \mathrm{mg}$ versus $800 \mathrm{mg}$ daily dose of imatinib with a relative risk reduction of $61 \%$ in patients who received the $800 \mathrm{mg}$ daily dose. Some $p d g f r-a$ mutations are more responsive to imatinib than others, such as mutations in exon 12 or 14 [40]; however the most common mutation in pdgfr-a is in exon 18 which is resistant to imatinib in in vitro studies [40].

Secondary imatinib resistance is a phenomenon of GIST progression after a period of tumor control [41]. Patients who have progression of disease within the first 2 months of imatinib therapy are thought to have primary imatinib resistance. Patients with disease previously sensitive to imatinib who have evidence of disease progression may have outgrowth of tumor with secondary mutations in either the c-kit or pdgfr-a or development of resistance through different mechanisms. Gene amplification of the activated tyrosine kinase, loss altogether of C-KIT or PDGFR-A expression, interference with imatinib binding to the adenosine-triphosphate (ATP)binding site in the kinase and increased clearance of imatinib are some proposed mechanisms of secondary imatinib resistance [41]. Some of the mutations in $c$-kit associated with secondary resistance were discovered through molecular genotyping of tumor samples and in vitro modeling of sensitivity to kinase inhibition [38]. Mutations in exon 13 or 14 that encode the ATP binding pocket of C-KIT are the most frequently observed secondary mutations; the V654A mutation in exon 13 is a common secondary mutation. Mutations in exon 17 that encodes the activation loop of C-KIT have been seen as well [38]. Interestingly, a study performed by Heinrich, et al. [38] observed secondary mutations more commonly in GISTs that had a primary exon $11 \mathrm{c}$-kit mutation compared to tumors that had a primary exon $9 c$-kit mutation (73\% vs. $19 \% P=0.0003)$. In the same study, there were only four patients with primary $p d g f r-a$ mutations, one of which had a primary exon 12 mutation and a secondary exon 18 mutation. Not surprisingly, the GISTs with primary exon 18 mutations did not develop secondary mutations, supporting in vitro studies that GISTs with $p d g f r-a$ exon 18 mutations are primarily resistant to imatinib. Patients found to have secondary resistance to imatinib may be managed by increasing the dose of imatinib (if not previously done), switched to second-line treatment using sunitinib or enrolled in a clinical trial.

\section{Sunitinib Use in GIST}

Sunitinib malate is a direct inhibitor of C-KIT, PDGFR, vascular endothelial growth factor (VEGF)-1, VEGF-2, VEGF-3, Fms-like tyrosine kinase-3 (FLT3), and the receptor encoded by proto-onogene ret. Sunitinib is currently approved for use in advanced GIST that is imatinib resistant or in patients with advanced GIST who are not able to tolerate imatinib. A multi-center phase III trial evaluating sunitinib for these indications enrolled 312 patients with advanced GIST between 2003 and 2005 who were previously on imatinib and either could not tolerate the medication or in which disease progressed [42]. Two-hundred and seven patients were randomized to receive sunitinib $50 \mathrm{mg}$ daily for 4 weeks followed by a 2-week break per cycle of treatment, and 105 patients received placebo. The trial was unblinded early due to an interim analysis revealing a dramatic effect of sunitinib versus placebo in patients after two cycles. Median time to tumor progression was 26 weeks in patients receiving sunitinib and 6 weeks in the placebo group. Even though cross-over from placebo to sunitinib was allowed upon progression, overall survival was better in the group initially assigned to sunitinib (hazard ratio (HR) $0.49, P=0.007$ ), though the median survival had not yet been reached since the majority of the patients in the sunitinib group were living at the time of interim analysis.

\section{Dosing of Sunitinib}

The currently approved dose for sunitinib as second-line therapy in advanced GIST is a 6-week cycle with $50 \mathrm{mg}$ daily for 4 weeks followed by 2 weeks off ( $4 / 2$ schedule). A phase II study recently evaluated whether continuous daily dosing at a lower dose of $37.5 \mathrm{mg}$ daily would be potentially as efficacious and less toxic than the $4 / 2$ schedule [43]. The median progression-free survival was 34 weeks and the median overall survival at the time of analysis was 107 weeks, which the authors noted to be comparable to the phase III study reported by Demetri, et al. However, the phase II study of continuous daily dosing of sunitinib was not a randomized comparative trial. The adverse effects reported in the study evaluating the $4 / 2$ schedule and the study evaluating continuous daily dosing were comparable. Further discussion of adverse effects of sunitinib will be discussed below. Due to the similar efficacy and adverse effect profile, the authors concluded that the continuous daily dosing could be seen as an acceptable alternative to the currently recommended $4 / 2$ schedule, though the study was not designed to evaluate equivalence or superiority compared to the approved dose and schedule.

\section{Metabolism of Sunitinib}

Like imatinib, sunitinib is also metabolized by the CYP3A4 enzyme, and therefore potential drug interactions that could increase or decrease the serum levels of this medication are the same as those seen with imatinib. One specific recommendation noted in the NCCN guidelines addresses the concurrent administration of sunitinib with a medication that induces the CYP3A4 enzyme, thereby lowering the serum concentration of sunitinib. In this situation, the dose of sunitinib can be titrated to a maximum of $87.5 \mathrm{mg}$ in the 4 weeks on, 2 weeks off schedule as long as patients are carefully monitored for toxicities [1].

\section{Adverse Effects of Sunitinib}

Adverse events associated with sunitinib are relatively common, but usually are only mild to moderate. Fatigue, diarrhea, and skin discoloration were the most common non-hematologic adverse events seen in the phase III study comparing sunitinib to placebo, with the majority of these being grades 1-2 [42]. Nausea, decreased appetite, hand-foot syndrome, and rash were also seen in more than $10 \%$ of patients, though again mostly at grade 1 or 2 [42]. In the study evaluating continuous daily dosing, diarrhea, fatigue, hypertension, and nausea were the most common adverse events [43]. Anemia and neutropenia are common hematologic adverse events seen in both $4 / 2$ schedule and the continuous daily schedule $[42,43]$. However, in the trial comparing sunitinib to placebo, anemia occurred in $62 \%$ of patients receiving sunitinib compared to $60 \%$ of patients receiving placebo, illustrating that some adverse effects associated with treatment are due to tumor burden [42]. Neutropenia, on the other hand, was seen in $51 \%$ of patients who received sunitinib and only $4 \%$ of patients who received placebo [42]. Hypertension is a side effect that has been typically seen in patients receiving sunitinib for renal cell carcinoma, but is less commonly seen in patients receiving sunitinib for GIST, despite similar dosing [44]. A 
study evaluating the toxicities of sunitinib in patients with renal cell carcinoma versus non-renal cell carcinoma patients revealed a $25.9 \%$ incidence of any grade hypertension in renal cell patients versus a $19.6 \%$ incidence in patients on sunitinib for other reasons. This discrepancy may be related to involvement of the kidney in renal cell carcinoma or prior nephrectomy that may predispose patients to hypertension. $\mathrm{CHF}$ is another more serious adverse event that has been found to occur in association with sunitinib. In the Phase I/II studies of sunitinib, CHF was detected in $8 \%$ of 75 patients evaluated. Almost $50 \%$ of 32 patients who received the approved dose of sunitinib had a decrease in their ejection fraction by at least $10 \%$ [45]. Hypothyroidism has recently been reported as well; a prospective, observational cohort study detected an abnormal thyroid stimulating hormone (TSH) level in $62 \%$ of patients on suninitb [46]. Therefore, the NCCN task force recommends following TSH levels every 3 6 months as long as patients are being treated with sunitinib [1].

\section{Resistance to Sunitinib}

Sunitinib malate is a small-molecule tyrosine kinase inhibitor, similar to imatinib, though selective for KIT, PDGFRA, PDGFRB, all three isoforms of VEGFR, FLT3, CSF-1R, and RET. Sunitinib has been shown to be effective against certain mutations in GISTs that are resistant to imatinib, whether through primary or secondary resistance.

A continuation trial of sunitinib in 97 patients with metastatic imatinib-resistant or imatinib-intolerant GIST assessed the mutational status in 78 tumor specimens from enrolled patients [38]. After 6 months, partial response or stable disease rate observed in patients with primary mutations in $c$-kit exon 9, $c$-kit exon 11, and wild-type $c$-kit/pdgfr tumors was $58 \%, 34 \%$, and $56 \%$, respectively. No clinical benefit (defined as partial response or stable disease) was observed for the four patients with mutations in $p d g f r-a$. Progression-free survival and overall survival were significantly longer for patients with primary $c$-kit exon 9 mutations and wild-type tumors compared to those with $c$-kit exon 11 mutations. Median overall survival in patients with an exon 9 mutation or a wild-type tumor was 26.9 months $(P=0.12)$ and 30.5 months $(P=0.132)$, compared to only 12.3 months in patients with an exon 11 mutation. The fact that sunitinib was more effective in tumors with exon 9 mutations compared to those with exon 11 mutations is not an indication that exon 11 mutant GIST is resistant to sunitinib, but is a testament that secondary mutations in primary $c$-kit exon 11 mutated GIST leading to imatinib resistance also confer resistance to sunitinib. Median progression-free survival in patients with secondary c-kit mutations receiving sunitinib was significantly longer in those with a mutation in exon 13 or 14 of $c$-kit as compared to those patients with an exon 17 or $18 c$-kit mutation (7.8 months vs. 2.3 months, $P=0.0157)$.

In vitro analyses were done on cell lines from 78 tumor specimens from the studied patients to evaluate the potency of sunitinib against certain mutations [38]. Sunitinib was found to be potent in inhibiting the activity of the receptor in "wild-type" tumors and in tumors containing $c$-kit exon 11 V560D or exon 9 mutations. Tumors with $c$-kit double mutants (those tumors that developed secondary mutations) with the second mutation in the drug/ATP binding site of the receptor (exons 13 and 14) were potently inhibited by sunitinib, but tumors with secondary mutations observed in the activation loop (exons 17 and 18) were resistant to sunitinib. Sunitinib was also found to be effective at inhibiting phosphorylation (activation) of wild-type PDGFR-A or PDGFR-A with the V561D mutation. However, the most common PDGFR-A mutation in GIST, the D842V mutation in exon 18 , showed resistance to sunitinib.

A randomized trial of sunitinib versus imatinib as first-line treatment in patients with primary mutation in exon 9 of $c$-kit or with wild-type GIST has not been performed and would be difficult to complete because of the infrequency of primary $c$-kit exon 9 mutant GIST. However, the study reported by Heinrich, et al. [38] that evaluated in vitro activity of sunitinib and imatinib in tumors with different activating mutations demonstrated that sunitinib was more potent than imatinib in inhibiting growth of some of the GISTs harboring mutations in exon 9 of $c$-kit. Further study needs to be done to determine whether mutational analysis should be an important first step in determining the best treatment for patients diagnosed with advanced GIST. Currently, imatinib is the first-line agent for all patients diagnosed with advanced or metastatic GIST regardless of tumor mutational status, but an interesting question to investigate would be whether newly diagnosed patients with potentially imatinib-resistant mutations would benefit from first-line treatment using sunitinib or another tyrosine-kinase inhibitor.

\section{CONCLUSION}

Approximately 4,000-6,000 patients are diagnosed with GIST in the US yearly and less than one-half have advanced unresectable tumors or metastasis. For patients with localized, resectable GIST, complete resection is curative in the majority but risk stratification schemes provide important prognostic information. Prior to the introduction of small molecule tyrosine kinase inhibitor therapy, advanced or metastatic GIST was a rapidly fatal disease. Following the development of tyrosine-kinase inhibitor therapy, this rare but lethal sarcoma has become manageable and even a chronic condition in many patients. Imatinib $400 \mathrm{mg}$ daily is the recommended treatment for patients with unresectable or metastatic GIST with an expected tumor response rate of about $50 \%$, stable disease rate of about $35 \%$ and primary refractory disease to imatinib in about $15 \%$ of patients. In patients with tumor progression on $400 \mathrm{mg}$ daily of imatinib, control of disease may be achieved in about one-third of patients after increasing the dose to a total of $600-800 \mathrm{mg}$ daily. This maneuver seems to be more likely to control tumors that do not harbor a primary mutation in exon 11 of $c$-kit. Sunitinib is effective in the treatment of GIST and is approved for use after failure of or intolerance to imatinib. Many of the patients with advanced GIST treated with imatinib and/or sunitinib will develop drug-resistant tumors and are in desperate need of alternative treatments. As we learn more about the mutations associated with the pathogenesis of GIST, including the secondary mutations that develop while on firstline imatinib therapy or secondary therapy with sunitinib, with further refinement of the techniques to genotype GIST biopsies and development of small molecules to overcome the effects of secondary mutations in kinases, future therapy may be tailored to the individual patient based on mutational profile of the GIST. A realistic hope is that patients and clinicians will see advanced GIST turned from a fatal cancer into a long-term chronic disease.

\section{REFERENCES}

1. Demetri GD, von Mehren M, Antonescu CR, et al.: NCCN Task Force report: Update on the management of patients with gastrointestinal stromal tumors. J Natl Compr Canc Netw 2010;8:S1-S41.

2. Nilsson B, Bumming P, Meis-Kindblom JM, et al.: Gastrointestinal stromal tumors: The incidence, prevalence, clinical course, and prognostication in the preimatinib mesylate era - A population-based study in western Sweden. Cancer 2005;103:821829.

3. Blanke CD, Demetri GD, von Mehren M, et al.: Long-term results from a randomized phase II trial of standard- versus higher-dose imatinib mesylate for patients with unresectable or metastatic gastrointestinal stromal tumors expressing KIT. J Clin Oncol 2008;26:620-625. 
4. Reith JD, Goldblum JR, Lyles RH, et al.: Extragastrointestinal (soft tissue) stromal tumors: An analysis of 48 cases with emphasis on histologic predictors of outcome. Mod Pathol 2000;13:577-585.

5. Miettinen M, Sobin LH, Lasota J: Gastrointestinal stromal tumors of the stomach: A clinicopathologic, immunohistochemical, and molecular genetic study of 1765 cases with long-term follow-up. Am J Surg Pathol 2005;29:52-68.

6. DeMatteo RP, Lewis JJ, Leung D, et al.: Two hundred gastrointestinal stromal tumors: Recurrence patterns and prognostic factors for survival. Ann Surg 2000;231:51-58.

7. Heinrich MC, Corless CL, Demetri GD, et al.: Kinase mutations and imatinib response in patients with metastatic gastrointestinal stromal tumor. J Clin Oncol 2003;21:4342-4349.

8. Hirota S, Ohashi A, Nishida T, et al.: Gain-of-function mutations of platelet-derived growth factor receptor alpha gene in gastrointestinal stromal tumors. Gastroenterology 2003;125: 660-667.

9. Pantaleo MA, Astolfi A, Di Battista M, et al.: Insulin-like growth factor 1 receptor expression in wild-type GISTs: A potential novel therapeutic target. Int J Cancer 2009;125:29912994.

10. Braconi C, Bracci R, Bearzi I, et al.: Insulin-like growth factor (IGF) 1 and 2 help to predict disease outcome in GIST patients. Ann Oncol 2008;19:1293-1298.

11. Andersson J, Sihto H, Meis-Kindblom JM, et al.: NF1-associated gastrointestinal stromal tumors have unique clinical, phenotypic, and genotypic characteristics. Am J Surg Pathol 2005;29: $1170-1176$.

12. Miettinen M, Fetsch JF, Sobin LH, et al.: Gastrointestinal stromal tumors in patients with neurofibromatosis 1: A clinicopathologic and molecular genetic study of 45 cases. Am J Surg Pathol 2006;30:90-96.

13. Agarwal R, Robson M: Inherited predisposition to gastrointestinal stromal tumor. Hematol Oncol Clin North Am 2009;23:113

14. Gold JS, Gonen M, Gutierrez A, et al.: Development and validation of a prognostic nomogram for recurrence-free survival after complete surgical resection of localised primary gastrointestinal stromal tumour: A retrospective analysis. Lancet Oncol 2009;10:1045-1052.

15. Edmonson JH, Marks RS, Buckner JC, et al.: Contrast of response to dacarbazine, mitomycin, doxorubicin, and cisplatin (DMAP) plus GM-CSF between patients with advanced malignant gastrointestinal stromal tumors and patients with other advanced leiomyosarcomas. Cancer Invest 2002;20:605-612.

16. Dematteo RP, Heinrich MC, El-Rifai WM, et al.: Clinical management of gastrointestinal stromal tumors: Before and after STI-571. Hum Pathol 2002;33:466-477.

17. Berthet B, Sugarbaker TA, Chang D, et al.: Quantitative methodologies for selection of patients with recurrent abdominopelvic sarcoma for treatment. Eur J Cancer 1999;35:413-419.

18. Eilber FC, Rosen G, Forscher C, et al.: Surgical resection and intraperitoneal chemotherapy for recurrent abdominal sarcomas. Ann Surg Oncol 1999;6:645-650.

19. Hirota S, Isozaki K, Moriyama Y, et al.: Gain-of-function mutations of c-kit in human gastrointestinal stromal tumors. Science 1998;279:577-580.

20. Joensuu H, Roberts PJ, Sarlomo-Rikala M, et al.: Effect of the tyrosine kinase inhibitor STI571 in a patient with a metastatic gastrointestinal stromal tumor. N Engl J Med 2001;344:10521056.

21. Verweij J, Casali PG, Zalcberg J, et al.: Progression-free survival in gastrointestinal stromal tumours with high-dose imatinib: Randomised trial. Lancet 2004;364:1127-1134

22. Blanke CD, Rankin C, Demetri GD, et al.: Phase III randomized, intergroup trial assessing imatinib mesylate at two dose levels in patients with unresectable or metastatic gastrointestinal stromal tumors expressing the kit receptor tyrosine kinase: S0033. J Clin Oncol 2008;26:626-632.

23. Zalcberg JR, Verweij J, Casali PG, et al.: Outcome of patients with advanced gastro-intestinal stromal tumours crossing over to a daily imatinib dose of $800 \mathrm{mg}$ after progression on $400 \mathrm{mg}$ Eur J Cancer 2005;41:1751-1757.

24. Eisenberg BL, Harris J, Blanke CD, et al.: Phase II trial of neoadjuvant/adjuvant imatinib mesylate (IM) for advanced primary and metastatic/recurrent operable gastrointestinal stromal tumor (GIST): Early results of RTOG 0132/ACRIN 6665. J Surg Oncol 2009;99:42-47.

25. McAuliffe JC, Hunt KK, Lazar AJ, et al.: A randomized, phase II study of preoperative plus postoperative imatinib in GIST: Evidence of rapid radiographic response and temporal induction of tumor cell apoptosis. Ann Surg Oncol 2009;16:910-919.

26. Andtbacka RH, Ng CS, Scaife CL, et al.: Surgical resection of gastrointestinal stromal tumors after treatment with imatinib. Ann Surg Oncol 2007;14:14-24.

27. Hohenberger P, Oladeji O, Licht T, et al.: Neoadjuvant imatinib and organ preservation in locally advanced gastrointestinal stromal tumors (GIST. J Clin Oncol 2009;27:548s (abstract 10550).

28. Hecker A, Hecker B, Bassaly B, et al.: Dramatic regression and bleeding of a duodenal GIST during preoperative imatinib therapy: Case report and review. World J Surg Oncol 2010;8: 47.

29. Saied GM, Kensarah AM: Six months neoadjuvant imatinib improves resectability potential of gastric stromal tumors in Egyptian patients. Int J Surg 2010;8:105-108.

30. Demetri GD, Wang Y, Wehrle E, et al.: Imatinib plasma levels are correlated with clinical benefit in patients with unresectable/ metastatic gastrointestinal stromal tumors. J Clin Oncol 2009; 27:3141-3147.

31. Blay JV, Le Cesne A, Ray-Coquard I, et al.: Prospective multicentric randomized phase III study of imatinib in patients with advanced gastrointestinal stromal tumors comparing interruption versus continuation of treatment beyond 1 year: The French Sarcoma Group. J Clin Oncol 2007;25:1107-1113.

32. Le Cesne A, Ray-Coquard I, Bui BN, et al.: Continuous versus interruption of imatinib in responding patients with advanced GIST after three years of treatment: A prospective randomized phase III trial of the French Sarcoma Group. J Clin Oncol 2007;25:546s (abstract 10005).

33. Guilhot F: Indications for imatinib mesylate therapy and clinical management. Oncologist 2004;9:271-281.

34. Ganjoo KN, Demetri GD, Jacobs C, et al.: Acute myeloid leukemia in patients with gastrointestinal stromal tumors treated with Gleevec. Leuk Lymphoma 2009;50:1882-1884.

35. Kerkela R, Grazette L, Yacobi R, et al.: Cardiotoxicity of the cancer therapeutic agent imatinib mesylate. Nat Med 2006;12: 908-916.

36. Debiec-Rychter M, Sciot R, Le Cesne A, et al.: KIT mutations and dose selection for imatinib in patients with advanced gastrointestinal stromal tumours. Eur J Cancer 2006;42:10931103.

37. Heinrich MC, Owzar K, Corless CL, et al.: Correlation of kinase genotype and clinical outcome in the North American Intergroup Phase III Trial of imatinib mesylate for treatment of advanced gastrointestinal stromal tumor: CALGB 150105 Study by Cancer and Leukemia Group B and Southwest Oncology Group. J Clin Oncol 2008;26:5360-5367.

38. Heinrich MC, Maki RG, Corless CL, et al.: Primary and secondary kinase genotypes correlate with the biological and clinical activity of sunitinib in imatinib-resistant gastrointestinal stromal tumor. J Clin Oncol 2008;26:5352-5359.

39. Debiec-Rychter M, Dumez H, Judson I, et al.: Use of c-KIT/ PDGFRA mutational analysis to predict the clinical response to imatinib in patients with advanced gastrointestinal stromal tumours entered on phase I and II studies of the EORTC Soft Tissue and Bone Sarcoma Group. Eur J Cancer 2004;40:689695.

40. Corless CL, Schroeder A, Griffith D, et al.: PDGFRA mutations in gastrointestinal stromal tumors: Frequency, spectrum and in vitro sensitivity to imatinib. J Clin Oncol 2005;23:53575364.

41. Maleddu A, Pantaleo MA, Nannini M, et al.: Mechanisms of secondary resistance to tyrosine kinase inhibitors in 
gastrointestinal stromal tumours (Review). Oncol Rep 2009;21: 1359-1366.

42. Demetri GD, van Oosterom AT, Garrett CR, et al.: Efficacy and safety of sunitinib in patients with advanced gastrointestinal stromal tumour after failure of imatinib: A randomised controlled trial. Lancet 2006;368:1329-1338.

43. George S, Blay JY, Casali PG, et al.: Clinical evaluation of continuous daily dosing of sunitinib malate in patients with advanced gastrointestinal stromal tumour after imatinib failure. Eur J Cancer 2009;45:1959-1968.
44. Zhu X, Stergiopoulos K: Wu S: Risk of hypertension and renal dysfunction with an angiogenesis inhibitor sunitinib: Systematic review and meta-analysis. Acta Oncol 2009;48:9-17.

45. Chu TF, Rupnick MA, Kerkela R, et al.: Cardiotoxicity associated with tyrosine kinase inhibitor sunitinib. Lancet 2007;370 2011-2019.

46. Desai J, Yassa L, Marqusee E, et al.: Hypothyroidism after sunitinib treatment for patients with gastrointestinal stromal tumors. Ann Intern Med 2006;145:660-664. 